\title{
WEIGHTED MORREY ESTIMATES FOR HAUSDORFF OPERATOR AND ITS COMMUTATOR ON THE HEISENBERG GROUP
}

\author{
JIANMIAO RUAN, DASHAN FAN AND QINGYAN WU
}

\begin{abstract}
In this paper, we study the high-dimensional Hausdorff operators, defined via a general linear mapping $A$, and their commutators on the weighted Morrey spaces in the setting of the Heisenberg group. Particularly, under some assumption on the mapping $A$, we establish their sharp boundedness on the power weighted Morrey spaces.
\end{abstract}

Mathematics subject classification (2010): 42B35, 46E30, 22E25.

Keywords and phrases: Hausdorff operator, commutator, Morrey space, weight.

\section{REFERENCES}

[1] D. R. Adams And J. Xiao, Morrey spaces in harmonic analysis, Ark. Mat. 50, (2012), 201-230.

[2] J. Alvarez, J. Lakey and M. Guzúan-Partida, Spaces of bounded $\lambda$-cenetral mean oscillation, Morrey Spaces, and $\lambda$-central Carleson measure, Collect. Math. 51, (2000), 1-47.

[3] H. ARAi AND T. MizuhaRA, Morrey spaces on spaces of homogeneous type and estimates for $\square_{b}$ and the Cauchy-Szegö projection, Math. Nachr. 185, (1997), 5-20.

[4] J. Chen, D. FAn AND J. LI, Hausdorff operators on function spaces, Chin. Ann. Math. Ser. B, 33, (2012), 537-556.

[5] J. Chen, D. Fan, X. Li And J. Ruan, The fractional Hausdorff operators on the Hardy spaces $H^{p}\left(\mathbf{R}^{n}\right)$, Anal. Math. 42, (2016), 1-17.

[6] J. ChEn, D. FAn And S. WANG, Hausdorff operators on Euclidean space, Appl. Math. J. Chinese Univ. Ser. B, 28, (2014), 548-564.

[7] J. Chen AND X. ZHU, Boundedness of multidimensional Hausdorff operators on $H^{1}\left(\mathbf{R}^{n}\right)$, J. Math. Anal. Appl. 409, (2014), 428-434.

[8] F. Chiarenza And M. Frasca, Morrey spaces and Hardy-Littlewood maximal function, Rend. Mat. Appl. 7, (1987), 273-279.

[9] X. T. Duong, J. Xiao And L. X. Yan, Old and new Morrey spaces with heat kernel bounds, J. Fourier Anal. Appl. 13, (2007), 87-111.

[10] G. Folland And E. Stein, Hardy Spaces on Homogeneous Groups, Mathematical Notes 28, Princeton University Press, Princeton, N.J., University of Tokyo Press, Tokyo, 1982.

[11] V. GuliEv, Two-weighted $L^{p}$-inequalities for singular integral operators on Heisenberg groups, Georgian Math. J. 1, 4 (1994), 367-376.

[12] R. HowE, On the role of the Heisenberg group in harmonic analysis, Bull. Amer. Math. Soc. 3, (1980), $821-843$.

[13] T. HYTÖNEN, C. PÉREZ AND E. RELA, Sharp reverse Hölder property for $A_{\infty}$ weights on spaces of homogeneous type, J. Funct. Anal. 263, (2012), 3883-3899.

[14] S. IndRATNO, D. MALdonAdo AND S. Silwal, A visual formalism for weights satisfying reverse inequalities, Expo. Math. 33, (2015), 1-29.

[15] Y. Kanjin, The Hausdorff operator on the real Hardy spaces $H^{p}(\mathbf{R})$, Studia Math. 148, (2001), $37-45$.

[16] A. Korányi And H. Reimann, Quasiconformal mappings on the Heisenberg group, Invent. Math. 80, (1985), 309-338.

[17] Y. KOMORI AND S. SHIRAI, Weighted Morrey spaces and a singular integral operator, Math. Nachr. 282, (2009), 219-231. 
[18] A. LeRner And E. Liflyand, Multidimensional Hausdorff operators on real Hardy spaces, J. Aust. Math. Soc. 83, (2007), 79-86.

[19] E. LifLyAnd, Open problems on Hausdorffoperators, Complex Analysis and Potential Theory, World Sci. Publ., Hackensack, NJ, (2007), 280-285.

[20] E. Liflyand, Boundedness of multidimensional Hausdorff operators on $H^{1}\left(\mathbf{R}^{n}\right)$, Acta Sci. Math. (Szeged), 74, (2008), 845-851.

[21] E. LifLYAND, Hausdorff operators on Hardy spaces, Eurasian Math. J. 4, (2013), 101-141.

[22] E. LifLYAND AND A. MiYACHI, Boundedness of the Hausdorff operators in $H^{p}$ spaces, $0<p<1$, Studia Math. 194 (2009), 279-292.

[23] E. Liflyand AND F. MóRICZ, The Hausdorff operator is bounded on the real Hardy space $H^{1}(\mathbf{R})$, Proc. Amer. Math. Soc. 128, (2000), 1391-1396.

[24] E. LiflyAND AND F. MóRICZ, Commutating relations for Hausdorff operators and Hilbert transforms on real Hardy space, Acta Math. Hungar. 97, (2002), 133-143.

[25] S. Lu, Y. Ding AND D. YAn, Singular integrals and related topics, World Scientific Publishing Company, Singapore, 2007.

[26] A. MiYACHI, Boundedness of the Cesàro operator in Hardy space, J. Fourier Anal. Appl. 10, (2004), 83-92.

[27] F. MóRICZ, Multivariate Hausdorff operators on the spaces $H^{1}\left(\mathbf{R}^{n}\right)$ and $\mathrm{BMO}\left(\mathbf{R}^{\mathrm{n}}\right)$, Anal. Math. 31, (2005), 31-41.

[28] C. B. Morrey, On the solutions of quasi-linear elliptic partial differential equations, Trans. Amer. Math. Soc. 43, (1938), 126-166.

[29] B. Muckenhoupt, Weighted norm inequalities for the Hardy maximal function, Trans. Amer. Math. Soc. 165, (1972), 207-226.

[30] J. RUAn AND D. FAn, Hausdorff operators on the power weighted Hardy spaces, J. Math. Anal. Appl. 433, (2016), 31-48.

[31] J. RUAN AND D. FAN, Hausdorff operators on the weighted Herz-type Hardy spaces, Math. Inequal. Appl. 19, (2016), 565-587.

[32] J. RUAN AND D. FAN, Hausdorff type operators on the power weighted Hardy spaces $H_{|\cdot| \alpha}^{p}\left(\mathbf{R}^{n}\right)$, Math. Nachr. 290, (2017), 2388-2400.

[33] J. RUAN, D. FAN AND Q. WU, Weighted Herz space estimates for Hausdorff operators on the Heisenberg group, Banach J. Math. Anal. 11, (2017), 513-535.

[34] W. SCHEMPP, Harmonic analysis on the Heisenberg nilpotent Lie group, with applications to signal theory, Longman Sci. and Tech. Pitman Research Notes in Math Sci. 147, Harlow, Essex, 1986.

[35] F. WEISZ, The boundedness of the Hausdorff operator on multi-dimensional Hardy spaces, Analysis (Munich), 24, (2004), 183-195.

[36] Q. WU AND D. FAN, Hardy space estimates of Hausdorff operators on the Heisenberg group, Nonlinear Anal. 164, (2017), 135-154.

[37] Q. WU AND Z. FU, Weighted p-adic Hardy operators and their commutators on p-adic central Morrey spaces, Bull. Malays. Math. Sci. Soc. 40, 2 (2017), 635-654.

[38] X. WU, Necessary and sufficient conditions for generalized Hausdorff operators and commutators, Ann. Funct. Anal., 6, (2015), 60-72. 